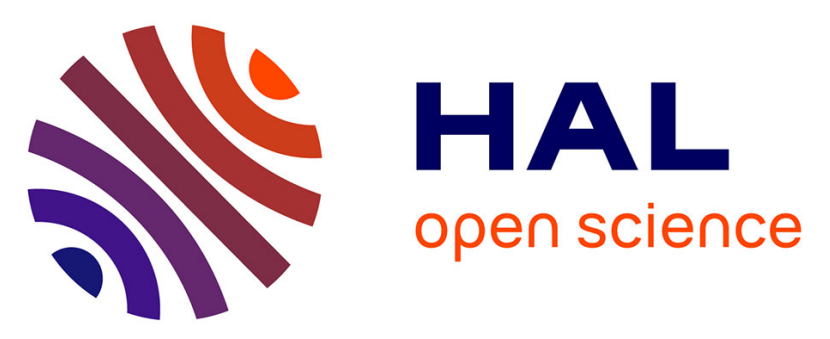

\title{
Cancers cutanés et bronchopulmonaire chez un viticulteur après expositions répétées à l'arsénite de soude
}

\author{
M. Kergresse, Pierre Rucay, C. Pajot, M. Guillier, Yves Roquelaure
}

\section{To cite this version:}

M. Kergresse, Pierre Rucay, C. Pajot, M. Guillier, Yves Roquelaure. Cancers cutanés et bronchopulmonaire chez un viticulteur après expositions répétées à l'arsénite de soude. Archives des Maladies Professionnelles et de L'Environnement, 2011, 72 (3), pp.281 - 284. 10.1016/j.admp.2011.04.007 . hal-03389869

\section{HAL Id: hal-03389869 \\ https://univ-angers.hal.science/hal-03389869}

Submitted on 21 Oct 2021

HAL is a multi-disciplinary open access archive for the deposit and dissemination of scientific research documents, whether they are published or not. The documents may come from teaching and research institutions in France or abroad, or from public or private research centers.
L'archive ouverte pluridisciplinaire HAL, est destinée au dépôt et à la diffusion de documents scientifiques de niveau recherche, publiés ou non, émanant des établissements d'enseignement et de recherche français ou étrangers, des laboratoires publics ou privés. 
Reçu le :

12 décembre 2010

Accepté le :

27 janvier 2011

\section{Cancers cutanés et bronchopulmonaire chez un viticulteur après expositions répétées à l'arsénite de soude}

\author{
Skin and lung cancers after repeated occupational exposure \\ with sodium arsenite in viticulture
}

M. Kergresse ${ }^{a, *, d}$, P. Rucay ${ }^{a, d}$, C. Pajot ${ }^{b}, M$. Guillier$^{c}$, Y. Roquelaure ${ }^{a, d}$

a Consultations de pathologies professionnelles, médecine E, CHU d'Angers, 4, rue Larrey 49933, Angers cedex 9, France

Service de dermatologie, CHU d'Angers, 4, rue Larrey 49933, Angers cedex 9, France

' ${ }^{\circ}$ Service de santé au travail, mutualité sociale agricole du Maine et Loire,

3, rue Charles-Lacretelle, Beaucouzé, 49938 Angers cedex 9, France

¿ UPRES EA 4336, laboratoire d'ergonomie et d'épidémiologie en santé au travail (LEEST),

$\checkmark$ UA InVS, IFR 132, Médecine E, CHU Angers, 4, rue Larrey, 49933 Angers, France

\section{Summary}

Arsenic is a metalloid whose soluble inorganic compounds are highly toxic. Sodium arsenite was used until 2001 as an antifungal in French viticulture. It has been classified as a carcinogen by the IARC in 1979. There is currently no efficient alternative.

The case reported is the story of that of a man aged 62, retired wine worker, a smoker, exposed to sodium arsenite (identified on the entrepreneur's invoice). In late winter he sprayed this type of product at the foot of the vines with a knapsack sprayer during five to 10 full days from 1966 to 1975 , and then he did it on 7 ha with a hose connected to a "tonne" each year until 1991, on approximately 7 ha. He was suffering from several squamous cell carcinomas since the beginning of the $80 \mathrm{~s}$, and then he was operated on (left pneumectomy) for lung squamous cell carcinoma on the left lower lobe (pT4N1 Mx) in 2006.

These two cancer sites are attributable to the use of sodium arsenite by presumption of origin. They were recognized in 2009, as compensable occupational diseases in the agriculture scheme. The possibility of chronic arsenic exposure in viticulture is unlikely in France since the campaign of eradication in 2006-2007. Due to the long time before the onset of cancers after arsenical exposure, cases may still appear among active workers or retirees. Information remains nevertheless necessary to occupational physicians, general practitionners, dermatologists and pulmonologists to fight against under-reporting such cancers. Finally, a postoperative professional

\section{Résumé}

L'arsenic est un métalloïde dont les composés inorganiques solubles ont une toxicité élevée. L'arsénite de soude, composé arsenical inorganique à l'état trivalent, a été utilisé jusqu'en 2001 comme antifongique en viticulture française. Il a été classé dans le groupe des agents cancérogènes avérés par le Centre international de recherche sur le cancer (CIRC) dès 1979. Il n'existe aucune substitution efficiente actuellement.

Le cas rapporté relate l'histoire d'un homme âgé de 62 ans, ouvrier viticole retraité, fumeur, exposé à l'arsénite de soude (dont le Pyralesca ${ }^{\circledR}$ identifié sur les factures du chef d'entreprise). Il a été atteint de kératoses actiniques profuses, et de plusieurs carcinomes épidermoïdes dès le début des années 80, puis opéré (pneumonectomie gauche) d'un carcinome bronchique épidermoïde lobaire inférieur gauche (pT4N1 Mx) en 2006. Ces deux localisations cancéreuses sont imputables à l'utilisation d'arsénite de soude par présomption d'origine. Elles ont été reconnues courant 2009, en maladies professionnelles indemnisables du régime agricole. En raison du long délai d'apparition des cancers après expositions arsenicales, des cas peuvent toujours apparaître, a posteriori, chez des salariés en activité, ou retraités. Bien qu'un risque d'intoxication aiguë subsiste du fait de restes de stock, la possibilité d'une exposition chronique à l'arsénite en viticulture est improbable en France depuis la campagne d'éradication de 2006-2007 où $97 \%$ des stocks ont été traités. Une information reste néanmoins nécessaire

\footnotetext{
* Auteur correspondant.

e-mail : mathieu.kergresse@live.fr
}

1775-8785X/\$ - see front matter @ $\odot 2011$ Elsevier Masson SAS. Tous droits réservés. 
(follow-up) follow-up could be considered in the agricultural scheme.

(c) 2011 Elsevier Masson SAS. All rights reserved.

Keywords: Sodium arsenite, Viticulture, Carcinogenesis, Occupational

L'arsenic est un métalloïde dont les composés inorganiques solubles ont une toxicité élevée. Les composés inorganiques dans lesquels l'arsenic est à l'état trivalent sont les dérivés les plus toxiques. II s'agit de l'arsénite de soude, de l'anhydride arsénieux et de l'acide arsénieux. Il est reconnu que l'arsenic peut induire un cancer cutané après ingestion prolongée ou des contacts cutanés répétés et qu'il peut également favoriser la survenue de cancers bronchopulmonaires [1,2]. Nous rapportons l'observation d'un patient qui présentait une double localisation cancéreuse cutanée et pulmonaire après expositions professionnelles répétées à des produits phytosanitaires contenant des composés inorganiques de l'arsenic.

\section{Observation}

Il s'agissait d'un patient âgé de 62 ans, ouvrier viticole à la retraite, fumeur avec un tabagisme évalué à 50 paquetsannées. Il avait une artériopathie oblitérante des membres inférieurs de stade I et était suivi en dermatologie depuis 25 ans en raison de kératoses actiniques profuses et de plusieurs carcinomes épidermoïdes. Pour deux d'entre eux, l'un était invasif, bien différencié, kératinisant au niveau du canthus interne droit et mesurant $1,2 \mathrm{~cm}$ et l'autre était infiltrant, moyennement différencié, mesurait $1,4 \mathrm{~cm}$ de diamètre et était situé sur le dorsum nasal. Leur exérèse chirurgicale avait été réalisée en 2004. En octobre 2006, il consulta pour hémoptysie et une pneumectomie gauche fut réalisée en décembre pour un carcinome bronchique épidermoïde lobaire inférieur gauche ( $\mathrm{pT}_{4} \mathrm{~N} 1 \mathrm{Mx}$ ). Un traitement complémentaire par chimiothérapie et radiothérapie avait alors complété la prise en charge.

Le service de consultations de pathologies professionnelles d'Angers fut alors sollicité pour enquête professionnelle dans le cadre de ce cancer bronchopulmonaire. L'enquête d'exposition identifiera parmi les phytosanitaires achetés par l'employeur et utilisés à l'époque (factures fournies), de nombreux fongicides et en particulier le Pyralesca ${ }^{\circledR}$ (arsénite de sodium) appliqué par le patient cinq à dix jours pleins à la fin de l'hiver, aux pieds des vignes. Ces applications étaient réalisées à l'aide d'un pulvérisateur à dos de 1966 à 1975, puis jusqu'en 1991, avec une lance reliée à une tonne tirée par un auprès des médecins du travail, médecins généralistes, dermatologues et pneumologues afin de lutter contre la sous-déclaration de ce type de cancers professionnels. Enfin, un suivi post-professionnel pourrait être envisagé en régime agricole.

(C) 2011 Elsevier Masson SAS. Tous droits réservés.

Mots clés : Arsénite de soude, Viticulture, Cancérogenèse, Professionnel

tracteur dépourvu de cabine (superficie traitée estimée à 7 ha par an). La préparation du produit était alors faite dans un hangar de l'entreprise.

L'examen clinique, le jour de la consultation, montre un patient en rémission du point de vue pulmonaire. On notait des kératoses actiniques infiltrées de la lèvre inférieure, de l'oreille gauche et du dos de la main gauche (fig. 1 et 2). La double localisation cancéreuse, présentée par le patient, $s^{\prime}$ inscrivait dans le tableau ${ }^{\circ}{ }^{10}$ des maladies professionnelles du régime agricole. Parmi la liste indicative des travaux entrant dans ce tableau, est mentionnée " toute manipulation ou emploi d'arsenic ou de ses composés minéraux, notamment lors des traitements anticryptogamiques de la vigne". Le délai de prise en charge de 40 ans pour le carcinome cutané

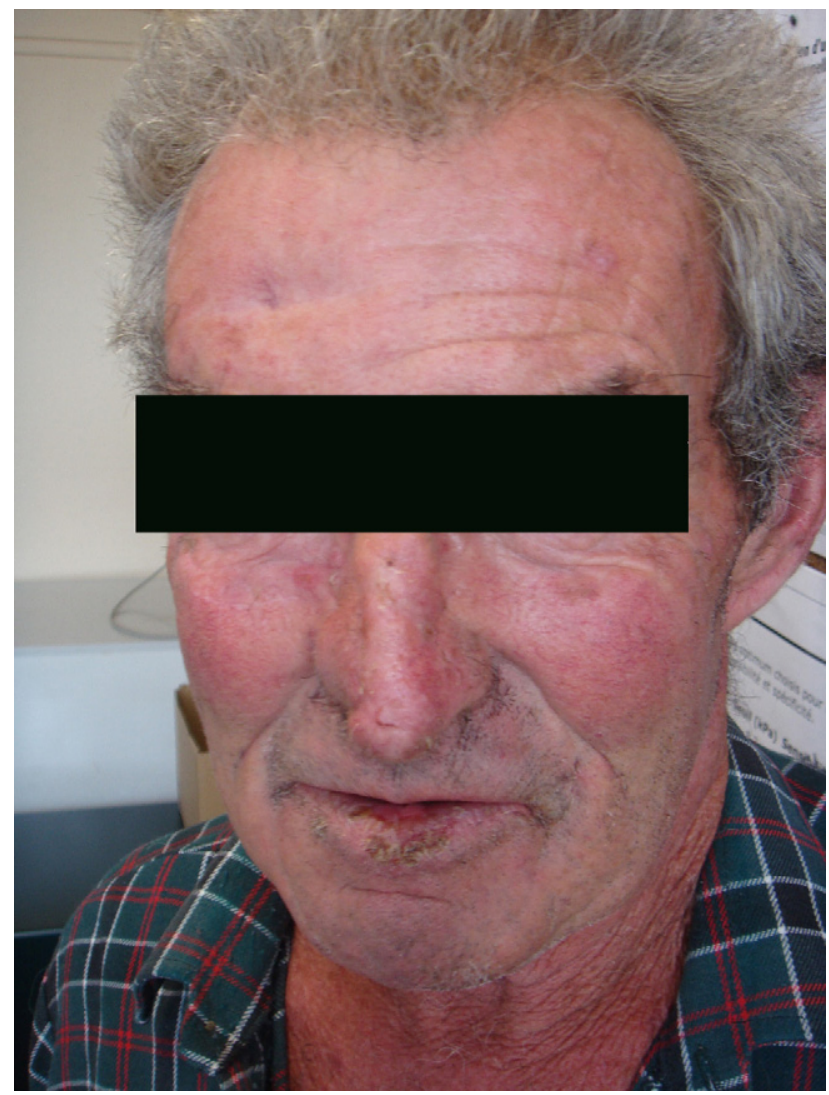

Figure 1. Kératose actinique de la lèvre inférieure et du nez. 


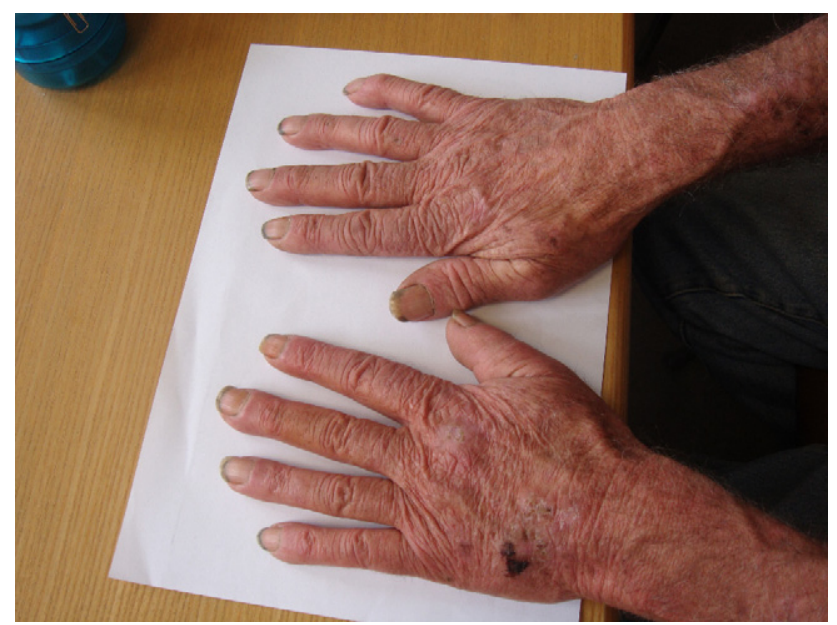

Figure 2. Kératose actinique infiltrée du dos de la main gauche.

épidermoïde était respecté ainsi que le délai de prise en charge de 40 ans et la durée d'exposition de dix ans pour le cancer bronchique primitif. Un formulaire de déclaration de maladie professionnelle a été rempli le jour de la consultation. La maladie professionnelle sera reconnue par l'organisme de sécurité sociale compétent.

\section{Discussion}

\section{Exposition au cancérogène}

L'arsenic a été utilisé dans de nombreuses applications en viticulture jusqu'en 2001 et les travailleurs ont pu y être exposés lors de la fabrication ou de l'utilisation de préparation arsenicale. L'usage de préparation contenant de l'arsénite de sodium dans la viticulture était à visée fongicide, il s'agit du dernier dérivé de l'arsenic employé en France en viticulture. Appliqué pendant les traitements d'hiver, il était efficace pour lutter contre l'ESCA, une affection qui conduit à la mort des ceps de vigne et entraîne à plus long terme, par remplacement des manquants, une hétérogénéité des parcelles. On ne dispose pas, à l'heure actuelle, de produit de substitution abordable financièrement et utilisable sur de grosses parcelles. La contamination et l'imprégnation des ouvriers viticoles lors de l'épandage de pesticides arsenicaux se faisaient par ingestion. En effet, l'arsénite est une substance minérale non volatile. De par leurs tailles, pour des raisons granulométriques, les gouttelettes générées par les dispositifs de pulvérisation étaient inhalées puis dégluties secondairement. De plus, la contamination était majorée par le contact de mains ou d'objets souillés avec la bouche ou par l'alimentation. Malgré les précautions, de nombreuses contaminations, lors de dispersions de bouillies, étaient inéluctables. Une étude, réalisée en 2000-2001, avait pu mettre en évidence, par biométrologie, qu'il n'existait pas de différence des niveaux d'imprégnation si le tracteur était équipé d'une cabine ou non et que le degré d'imprégnation augmentait tout au long du travail quotidien malgré les mesures de protection individuelle [3]. Des cas d'intoxications ont également été décrits suite à des consommations de vin contaminé par de l'arsénite de soude lorsque ce traitement était fait dans l'ignorance des pratiques professionnelles usuelles et après erreur de manipulation [4]. Les dérivés minéraux de l'arsenic sont classés dans le groupe des agents cancérogènes avérés par le Centre international de recherche sur le cancer (CIRC) depuis 1979 ainsi que par I'Union européenne. Les organes cibles pour lesquels la cancérogénicité de l'arsenic a été prouvée sont en premier lieu la peau et le poumon, l'association de ces deux cancers a déjà été citée par le passé notamment chez les viticulteurs du Beaujolais [5].

\section{Exposition à d'autres cancérogènes}

Le tabagisme marqué du patient a favorisé la genèse de son cancer bronchopulmonaire. D'un point de vue toxicologique c'est un élément majeur dans la genèse de ce cancer, l'exposition à l'arsenic pouvant être considérée de ce point de vue comme un cofacteur. Les rayonnements solaires (classés cancérogène de catégorie 1 selon le CIRC depuis 1992) ont également un rôle manifeste dans la survenue des antécédents dermatologiques du patient, mais cela n'intervient pas dans la procédure de réparation en maladie professionnelle indemnisable. Nous l'évoquerons par la suite.

\section{Campagne d'éradication de l'arsénite de soude}

L'absence de mesure de précaution efficace pour éviter l'imprégnation des travailleurs a abouti le 8 novembre 2001 à l'interdiction d'utilisation de l'arsénite de soude. II n'y a pas eu de mesure transitoire permettant l'écoulement des stocks. La campagne d'éradication de 2006 à 2007 a permis la collecte de 97 \% des stocks. L'exposition chronique à l'arsénite de soude ne se produira plus. Toutefois, du fait du délai d'apparition des pathologies cancéreuses, cette étiologie toxique est à garder en mémoire en ce qui concerne la réparation et le suivi post-exposition.

\section{Réparation}

Il est donc important de préciser que, juridiquement, le tabagisme et l'exposition solaire quotidienne du patient n'enlèvent en rien la présomption d'origine de l'arsénite de soude. En effet dans le système de tableaux de maladies professionnelles indemnisables, lorsque toutes les conditions de ce tableau sont remplies, qu'il s'agisse de la maladie, du délai et du type de travaux, la maladie professionnelle indemnisable est automatiquement reconnue par présomption d'origine $[6,7]$.

\section{Suivi post-exposition}

Dans la mesure du possible, lors de la cessation d'exposition à l'arsénite de soude, une attestation d'exposition au risque doit lui être remise au salarié. Cette attestation, remplie par 
l'employeur et le médecin du travail, précise notamment la nature, le niveau et la durée de l'exposition. Doivent y figurer les principales constatations médicales ainsi que les éventuels dosages biométrologiques effectués. En outre, une surveillance post-professionnelle pour l'exposition aux dérivés inorganique de l'arsenic serait souhaitable comme elle est prévue par le code de la sécurité sociale (article D 461-25) et appliquée dans le régime général. Dans le régime général, cette surveillance n'est pas systématique, il appartient à l'assuré d'en faire la demande à son organisme de Sécurité sociale. Les modalités de cette surveillance sont également prévues ; il s'agit, s'il y a eu manipulation, d'un examen de la peau. S'il y a eu exposition aux vapeurs ou aux poussières, la surveillance consiste en un examen clinique et une radio pulmonaire tous les deux ans. Ces examens sont pris en charge à $100 \%$ par le fonds d'action sanitaire et social de la sécurité sociale. Le médecin traitant peut également, dans le régime général, décider d'examens supplémentaires avec accord préalable du médecin conseil pour prise en charge à $100 \%$.

\section{Conclusion}

Le cas présenté illustre la toxicité de l'arsénite de soude et la multiplicité de ses organes cibles. Le risque d'intoxication chronique et cancérogène de l'arsénite de soude tend à disparaître du fait de la collecte de 2006-2007. Néanmoins, le risque d'intoxication aiguë subsiste par conduite suicidaire ou accident de déconditionnement du fait de restes de stock. Le délai de prise en charge de 40 ans, oblige tout de même le corps médical à garder en mémoire cette étiologie de cancers cutanés ou bronchopulmonaire chez les travailleurs de la vigne d'avant 2001 actifs ou retraités.

\section{Remerciements}

Nous remercions particulièrement le $\mathrm{Dr} C$. Kiener pour son aide précieuse.

\section{Références}

[1] Garnier R, Poupon J., Villa A. Arsenic et dérivés inorganiques. EMC (Elsevier Masson SAS, Paris) toxicologie-pathologies professionnelles, 16-002-A-30, 2008.

[2] Testud F. Fongicides arsenicaux. In: Testud F, Grillet JP, editors. Produits phytosanitaires : intoxications aigües et risques professionnels. Paris: ESKA; 2007. p. 185-98.

[3] Grillet JP, Adjemian A, Bernadac G, et al. Arsenic exposure in the wine growing industry in ten French departments. Int Arch Occup Environ Health 2004;77(2):130-5.

[4] LeBouil A, Avenel-Audran M, Bourgeais A-M, et al. Intoxication chronique à l'arsenic. Ann Toxicol Anal 2001;13(3):182-5.

[5] Galy P, Touraine R, Brune J, et al. Les cancers bronchopulmonaires de l'intoxication arsenicale chronique chez les viticulteurs du Beaujolais. Lyon Med 1963;43:735-44.

[6] Charbotel B., Normand J.-C., Bergeret A. Cancers professionnels. Généralités. EMC (Elsevier Masson SAS, Paris) toxicologiepathologies professionnelles, 16-532-A-05, 2007.

[7] ED 835. Les maladies professionnelles, guide d'accès aux tableaux du régime général et du régime agricole. Disponible sur www.inrs.fr (consulté le og mars 2010). 